\title{
Influence of droplets coverage on the electrochemical response of planar microelectrodes and potential solving strategies based on nesting concept
}

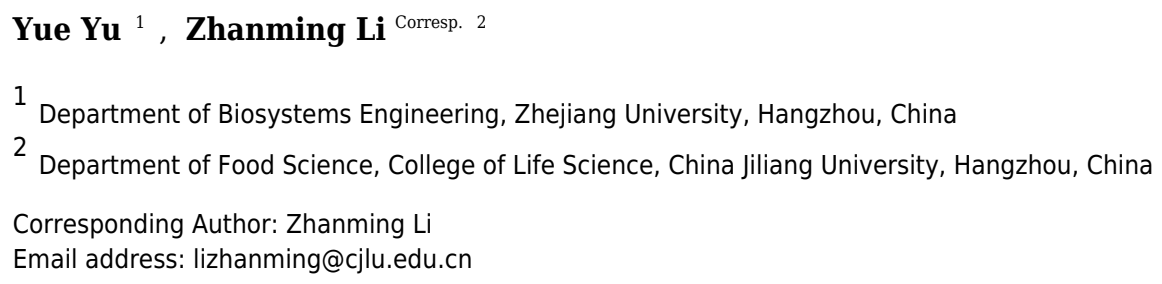

Recently, biosensors have been widely used for the detection of bacteria, virus and other toxins. Electrodes, as commonly used transducers, are vital part of electrochemical biosensors. The coverage of the droplets can change significantly based on the hydrophobicity of the microelectrode surface materials. In the present research, screenprinted interdigitated microelectrodes (SPIMs), as one type of planar microelectrodes, were applied to investigate the influence of droplets coverage on electrochemical response. Furthermore, three dimensional (3D) printing technology was employed to print smart devices with different diameters based on nesting concept. Theoretical explanations were proposed to elucidate the influence of the droplets coverage on the electrochemical response. 3D-printed ring devices were used to incubate the SPIMs and the analytical performances of the SPIMs were tested. According to the results obtained, our device successfully improved the stability of the signal responses and eliminated irregular signal changes to a large extent. Our proposed method based on nesting concept provide a promising method for the fabrication of stable electrochemical biosensors. We also introduced two types of electrode bases to improve the signal stability. 
1 Influence of droplets coverage on the electrochemical response of planar microelectrodes

2 and potential solving strategies based on nesting concept

3 Yue $\mathrm{Yu}^{1}$, Zhanming $\mathrm{Li}^{2 *}$

$4{ }^{1}$ Department of Biosystems Engineering, Zhejiang University, Hangzhou 310058, China.

5 2Department of Food Science, College of Life Science, China Jiliang University, Hangzhou 6310012 , China.

7

8 *Corresponding to: Dr. Zhanming Li, Phone: +86-571-86872488, Email:

9 lizhanming@cjlu.edu.cn

10 


\section{Abstract}

13 Recently, biosensors have been widely used for the detection of bacteria, virus and other toxins.

14 Electrodes, as commonly used transducers, are vital part of electrochemical biosensors. The

15 coverage of the droplets can change significantly based on the hydrophobicity of the

16 microelectrode surface materials. In the present research, screen-printed interdigitated

17 microelectrodes (SPIMs), as one type of planar microelectrodes, were applied to investigate the

18 influence of droplets coverage on electrochemical response. Furthermore, three dimensional (3D)

19 printing technology was employed to print smart devices with different diameters based on

20 nesting concept. Theoretical explanations were proposed to elucidate the influence of the

21 droplets coverage on the electrochemical response. 3D-printed ring devices were used to

22 incubate the SPIMs and the analytical performances of the SPIMs were tested. According to the

23 results obtained, our device successfully improved the stability of the signal responses and

24 eliminated irregular signal changes to a large extent. Our proposed method based on nesting

25 concept provide a promising method for the fabrication of stable electrochemical biosensors. We

26 also introduced two types of electrode bases to improve the signal stability.

\section{$27 \quad 1$ Introduction}

28 Recently, biosensors have been widely used for the detection of bacteria, virus and other

29 toxins. Biosensors are analytical systems composed of a biological sensing element and a

30 physical transducer. The transducer is designed for the conversion of biological information into

31 a detectable signal, such as proton concentration, absorption or reflectance, light emission, mass

32 changes, and so on (Hunt and Armani, 2010; Lepinay et al., 2014; Olaru et al., 2014; Thévenot et 
33 al., 2001; Van Dorst et al., 2010). Electrodes, as commonly used transducers, are vital part of

34 electrochemical biosensors. They are designed to transform the recognition of a biological

35 molecule into an easily quantifiable electrical signal (Gerard et al., 2002; Li et al., 2015b).

36 Electrodes with small dimensions, commonly referred to as microelectrodes, can maximize the

37 signal change, reduce the response time and work in a two-electrode system, thus benefiting the

38 fabrication and performance of electrochemical biosensors (Huey et al., 2012; Park and Beskok,

39 2008). Microelectrodes exhibit more accurate electrochemical response to low concentrations of

40 electro-active species in solution than large planar electrodes. Excellent flexibility and cycling

41 stability also promise potential applications in lab-on-a-chip systems (Ch et al., 2006; Huang et

42 al., 2009; Ueno et al., 2005). literatures (Bernalte et al., 2011; Taleat et al., 2014; Valentini et al., 2014; Wang et al., 2012; Zhu et al., 1998). The sensitivity and the signal-to-noise ratio of planar microelectrode can be influenced by many factors, such as the diameter of the electrode, surface coverage, electrode geometry and the electroactivity of analytes (Brett and Thiemann, 2002; Kostecki et al., 2000; Liu et al., 2014; Xu et al., 2004; Zhu et al., 1998). The droplets exhibit different coverage on the microelectrode surface, depending on the hydrophobicity of the microelectrode surface which may trigger irregular signal change. The commonly-used incubation methods for planar microelectrodes include dropping and immersing coatings. The influence of droplets coverage which is important but less concerned, should be evaluated when dropping coating is used for 
53 incubation. Screen-printed interdigitated microelectrodes (SPIMs), as one type of planar

54 microelectrodes, which may integrate the merits of screen printed microelectrodes and

55 interdigitated microelectrodes, have been used to develop sensitive, rapid-responding, cost-

56 effective biosensors ( $\mathrm{Li}$ et al., 2015a). In this manuscript, we investigate the influence of droplets

57 coverage on the electrochemical response of SPIMs.

58 Three-dimensional (3D) printed devices have captured much attention in many fields,

59 including food safety and analysis (Sun et al., 2015; Wei et al., 2015; Xing et al., 2015). As we

60 know, many birds build nests to incubate their eggs and raise their young in a protective

61 environment (Vilé et al., 2015). In order to further analyze the influence of the droplets coverage

62 quantitatively, 3D printing technology was employed to print smart nest-like devices with

63 different diameters to keep the immobilization and detection within the devices based on nesting

64 concept. Both the theoretical analysis and our experimental results support our conclusion that

65 the droplets coverage has significant influence on the electrochemical response of biosensors.

66 Depending on the varying hydrophobic properties of the materials used to immobilize the

67 surface of the electrodes, the droplets can show gathering or dispersing properties (Costa et al.,

68 2015; Gryczan et al., 2015; Santoro et al., 2014). According to such principle, different materials

69 are developed to remodel the surface of the SPIMs to improve the stability of signal responses.

70 In the present research, ring devices around the detection area were designed to gather the

71 droplets. Silica gel was used as a mode sample to validate our methodology. 3D-printed ring

72 devices were used to incubate the SPIMs and the analytical performances of our device and 
73 method were also evaluated. Software can also be used to handle the signal stability by electrode

74 base design.

75

76

77

78

79

80

81

82

83

84

85

86

87

\section{Materials and methods}

\subsection{Reagents and apparatus}

E. coli O157:H7 (ATCC43889) was purchased from ATCC (Manassas, MD). Biotin-anti-E. coli antibodies was obtained from Meridian Life Science (Saco, ME) and dissolved in PBS solution $(\mathrm{pH}=7.4)$. Bovine serum albumin (BSA), streptavidin (SA) and protein A were purchased from Sangon Biotech (Shanghai, China). MacConkey agar, brain heart infusion (BHI) culture medium were purchased from Becton, Dickinson and Company (Sparks, NV). PBS solution containing $10 \mathrm{mM} \mathrm{K}{ }_{3} \mathrm{Fe}(\mathrm{CN})_{6} / \mathrm{K}_{4} \mathrm{Fe}(\mathrm{CN})_{6}$ (Sangon Biotech., Shanghai, China) was used for electrochemical measurements. Ultrapure water $(18.2 \mathrm{M} \Omega \mathrm{cm})$ was obtained from a Millipore Milli-Q purification system (Merck Millipore, MA).

The width of a finger and the gap between two fingers for SPIM (AIBIT Biotech Instrument, China) are both $200 \mu \mathrm{m}$. One pair of gold electrodes and two welding plates were prepared on ceramic base using screen-printed technology. Two electrodes were connected to the bonding pad, respectively. The electrode contained multiple conducting ring with different diameter and connected by conductive band. Fig S1 showed the details of the electrodes.

Electrochemical impedance spectroscopy (EIS) and cyclic voltammetry (CV) were performed using ZAHNER electrochemical station (Kronach, Germany). Photosensitive resin 
92 was purchased from DSM SOMOS Crop. (Somos imagine 8000, Elgin, IL). The devices with

93 different diameters $(2 \mathrm{r}=6,7,8$ and 9 millimeter) were printed by a 3D printer (Liantai 450 ,

94 Shanghai, China) with 0.01 millimeter precision.

95

96

97

98

99

100

101

102

103

104

105

106

107

108

109

\subsection{Experimental methods}

\subsubsection{Preparation of Bacterial Samples}

E. coli $\mathrm{O} 157: \mathrm{H} 7$ was grown in $\mathrm{BHI}$ culture medium at $37^{\circ} \mathrm{C}$ for $20 \mathrm{~h}$ to the stationary phase. The stationary-phase cultures were diluted to $10^{7}-10^{1} \mathrm{cfu} \cdot \mathrm{mL}^{-1}$ in $\mathrm{PBS}(\mathrm{pH} 7.4)$ and $100 \mu \mathrm{L}$ of the diluted solutions were transferred to MacConkey agar plates and incubated at $37{ }^{\circ} \mathrm{C}$ for $24 \mathrm{~h}$ for enumeration of colonies. At the same time, the dilutions containing approximately $10^{5}$ $\mathrm{cfu} \cdot \mathrm{mL}^{-1}$ of bacteria cells were prepared for evaluation of the proposed devices.

\subsubsection{Dropping and immersing coatings}

When the planar microelectrodes were incubated, the wide commonly used incubation methods are dropping and immersing coatings for the incubation of planar microelectrodes. Usually, the volume of dropping coatings is 10 to $50 \mu \mathrm{L}$, and the volume of immersing coating is more than $1000 \mu \mathrm{L}$ in order to cover the whole detection area. Generally, the droplet coverage varies due to the discrepancy of the SPIMs surface. Figure 1 presents the droplets coverage of electrode. The droplets coverage for the bare SPIM is significantly different, compared to that for the modified SPIM (Fig S2). 
110

Figure 1. Detection area (Droplets area) of the electrode.

Furthermore, the droplets coverage may be changed during the modification process. Several factors that might have caused this include surface tension, gravity, interface hydrophobicity and the mobility of the molecules can be the reasons. In this experiment, different treatment groups were set up as comparison. Treatment groups $\mathrm{A}$ and $\mathrm{C}$ were immersing coating and treatment groups PBS and B were dropping coating, respectively. We used $1500 \mu \mathrm{L}$ PBS solution containing $50 \mu \mathrm{L}$ protein A for coating $\left(0.5 \mathrm{mg} \mathrm{mL}^{-1}\right)$ in treatment group $\mathrm{A}, 50 \mu \mathrm{L}$ protein $\mathrm{A}\left(0.5 \mathrm{mg} \mathrm{mL}^{-1}\right)$ in treatment group $\mathrm{B}$, and $1500 \mu \mathrm{L}$ protein $\mathrm{A}(0.5 \mathrm{mg}$ $\mathrm{mL}^{-1}$ ) in treatment group C. The signal change was detected with EIS or CV techniques.

\subsubsection{Nesting concept for incubation}

In order to investigate the difference caused by the droplet coverage, we designed and printed nest-like devices with different diameters. The devices (Figure 2) possess an equivalent volume, but with different height and diameter $(2 \mathrm{r}=6,7,8$ and 9 millimeter). The volume for these nest-like devices was equivalent, which ensured the same concentration and quantity of targets. The devices were incubated with BSA solution $(2 \%, \mathrm{w} / \mathrm{w})$ before use in order to avoid the non-specific absorption. Two devices ( $2 r=6$ and 9 millimeter) were selected to perform the test to investigate the difference introduced by the multistep modification. The devices were used 
128

129

130

131

132

133

134

135

136

137

to incubate SPIMs with SA solution $\left(50 \mu \mathrm{L}, 0.5 \mathrm{mg} \mathrm{mL}^{-1}\right)$ for $45 \mathrm{~min}$ and then biotin-antibody was immobilized according to our previous research (Li et al., 2015a). After that, the bacteria solution was added into the devices to incubate the SPIMs and the difference caused by multistep modification was evaluated.

A

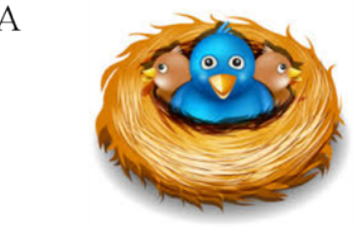

B

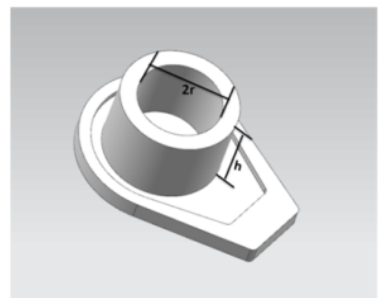

C

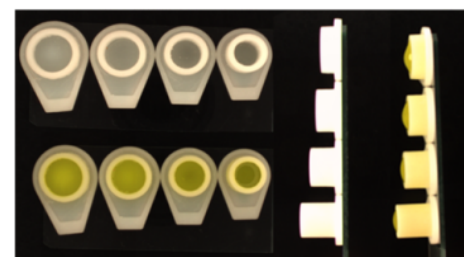

D

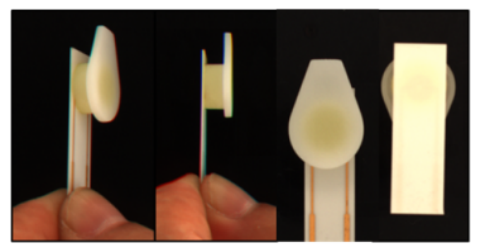

Figure 2. The 3D printed nest-like devices (B) with different height (h) and diameter ( $2 r=6,7,8$ and 9 millimeter) (C) and the photo of the incubation (D). The bird nest picture (A) was drew by Iconshock.

\subsubsection{D-printing ring devices}

In the present research, both resin and glass were used to investigate the dispersity of the target solution. A smart ring covered with silica gel was fabricated on the surface of the SPIM.

We used the nest-like devices with different diameters to cover the detection area and then use silica gel to coat the devices. The caps were removed after the gel was completely dry, in order to form a ring area. The ring can stay intact during the incubation and modification processes. In order to prove that the rings are intact, solutions with different color were added into the devices after each incubation and washing processes (Figure 3). The influence of $\mathrm{NaOH}$ solution was also investigated for the cleaning process. 

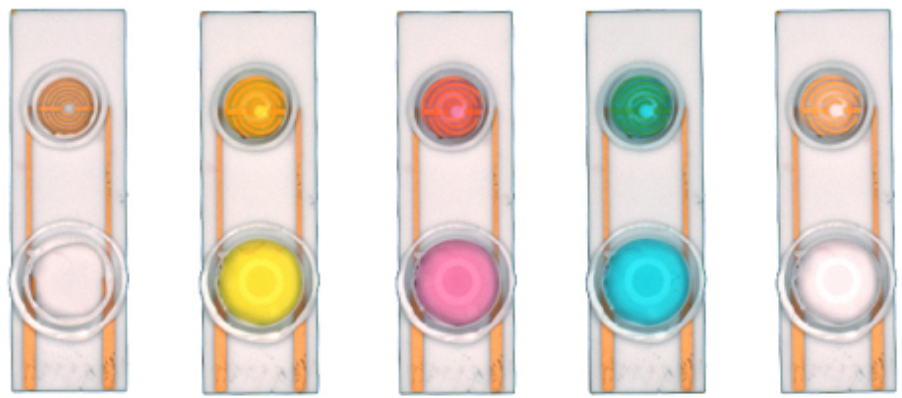

Figure 3. Silica gel ring on the surface of the SPIMs. Solution with different color were used to show the ring concept( from left to right: no solution, solution with $\mathrm{K}^{+}$, solution with $\mathrm{Co}^{3+}$, solution with $\mathrm{Cu}^{2+}$, ultrapure water).

(Figure 4). We selected two devices ( $2 r=6$ and 9 millimeter) to investigate the performance. The surface of these devices were also blocked with BSA solution to avoid non-specific absorption.

After that, these devices were fixed around of the detection area. The same amount of solution was washed with ultrapure water. The signal change was evaluated after the incubation.

A
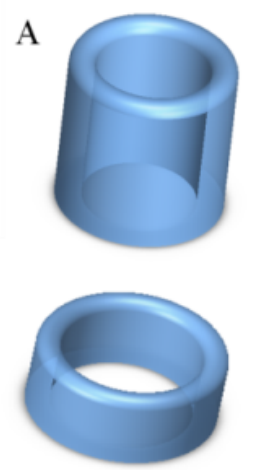
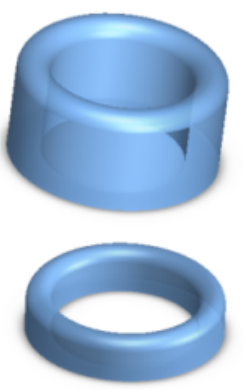

B

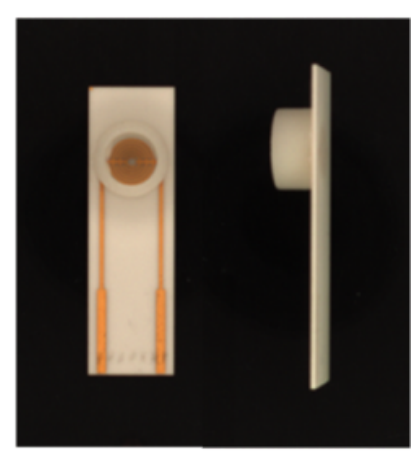

153

Figure 4. A) 3D printed ring devices with different diameters and B) SPIM with ring device to form a nest-like device.

\subsection{Statistical Analysis}

Experiments were conducted in triplicates for each concentration level of protein A and 
158 159 160 161 162

other materials. And bacteria cells were tested and the performance of the printed nest-like devices with different diameters were collected. Statistical analysis were conducted using SPSS 17.0. The biosensor responses were considered to show significant difference when P-value was less than 0.05 ( $95 \%$ confidence interval).

\section{Results and Discussion}

\subsection{Dropping and immersing coatings}

Four treatment groups were performed conducted and the application of PBS solution was used as a control (Figure 5). Performances of treatment A and B were significantly different and the signal change of treatment $\mathrm{B}$ was more obvious than that of treatment $\mathrm{A}$. Treatment $\mathrm{C}$ possessed more reactive molecules that can be captured on the surface of the devices in comparison with treatment $\mathrm{B}$. These results indicates that the signal changes are different between immersing and dropping coatings. Since the two methods required different amount of materials, it is not persuasive to conclude that the difference is caused by droplet coverage.

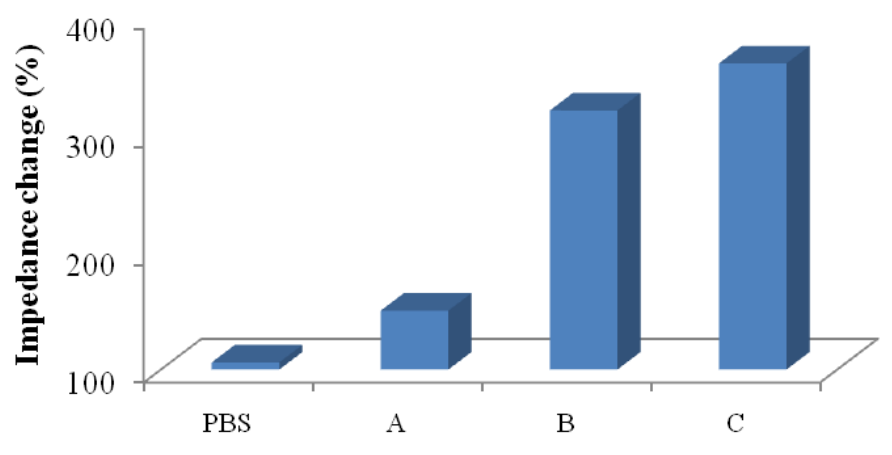

Different treatments

Figure 5. Immersing coating and dropping coating for the microelectrode modification. Treatment groups A and $\mathrm{C}$ were immersing coating and treatment groups PBS and B were dropping coating. Treatment group A:1500 $\mu \mathrm{L}$ PBS 
174

175

176

177

178

179

180

181

182

solution containing $50 \mu \mathrm{L}$ of protein $\mathrm{A}\left(0.5 \mathrm{mg} \mathrm{mL}^{-1}\right)$; treatment group B:50 $\mu \mathrm{L}$ of protein $\mathrm{A}\left(0.5 \mathrm{mg} \mathrm{mL}^{-1}\right)$; treatment group C: $1500 \mu \mathrm{L}$ of protein $\mathrm{A}\left(0.5 \mathrm{mg} \mathrm{mL}^{-1}\right)$.

\subsection{Performance of nest-like devices}

Considering the influence of the droplet coverage on the electrochemical response of SPIMs, smarter design of devices is necessary. Nest-like devices with different diameters were used for incubation (Figure 6). The results showed that there was significant difference between 9 millimeter and 6 millimeter devices $(\mathrm{p}>0.05)$ and the difference were not significant for other devices. According to the difference in the droplet coverage of these two devices, it can be concluded that the signal response is influenced by droplet coverage.

In order to investigate the influence of the multistep modification, we selected 6 and 9 millimeters devices to perform the test for bacteria detection (Figure 7). Treatment group A was prepared according to the signal change after SA modification. The results indicated that there was significant difference between the devices $(p>0.05)$. Treatment group B (Figure 7) was prepared according to the signal change of the bacteria incubation after the multistep modification. The difference between the results collected from these two treatment groups did not disappear. Due to the large dimension of the bacteria cells, not every site was occupied by the bacteria cell. However, only effective absorption can introduce signal response. From the results we concluded that the influence of droplets coverage was not disappeared by large dimension of cells. Therefore, it is clear that the droplet coverage is an influencing factor for electrochemical response. 


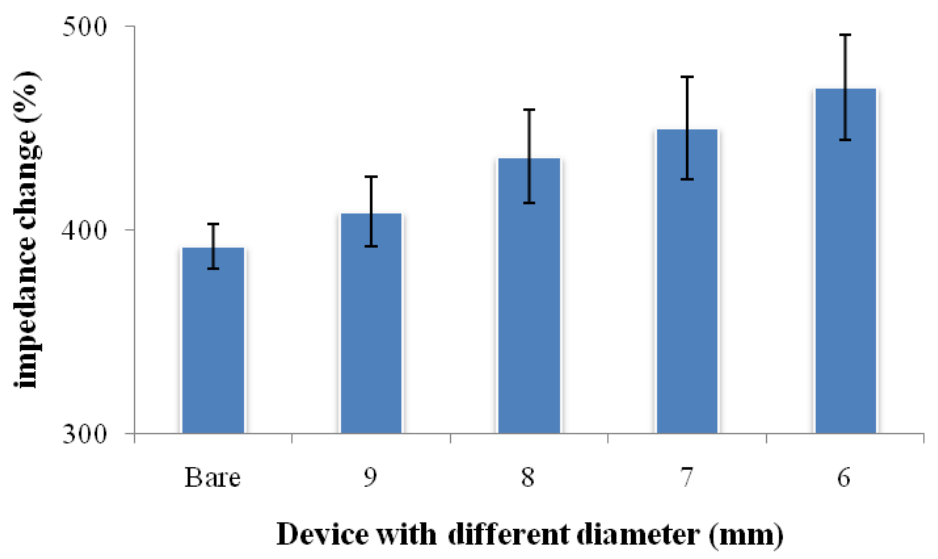

194

Figure 6. Signal change of the printed nest-like devices with different diameters for protein immobilization. Bare was a SPIM without printed devices.

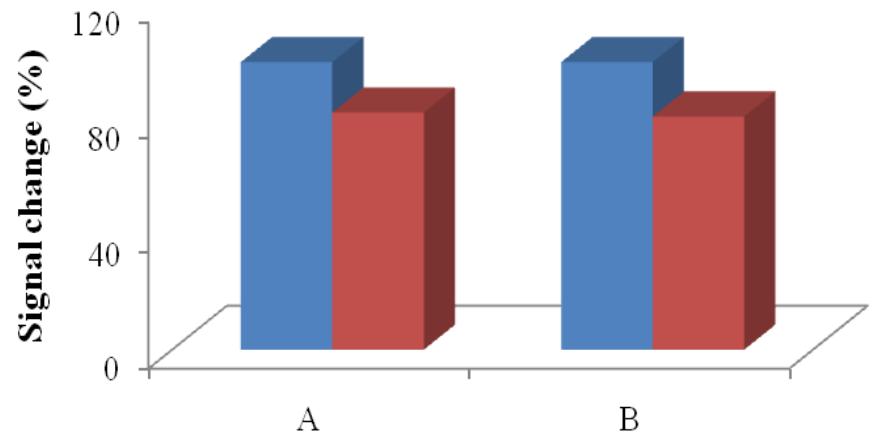

Different treatments

197

198

199

200

201

202

203

204

205

Figure 7. 3D printed nest-like devices ( 6 and 9 millimeters) for the test. Treatment group A was the performance after SA modification. Treatment group B was the performance of the bacteria incubation, after the multi-step modification.

\subsection{Performance of silica gel ring}

Resin and glass were used to investigate the dispersity of the target solution. The solution coverage were different due to the different hydrophobicity of these two materials. However, the surface constructed by these two materials was completely (or partly) damaged when washing solution $(\mathrm{NaOH}, 1 \mathrm{M})$ was used for SPIM regeneration. In order to deal with this situation, we 
206 firstly designed a ring was prepared on the surface of SPIM using silica gel. The results indicated

207 that the signal was suppressed significantly compared to the treatment groups without the device,

208 considering that more molecules were immobilized effectively (Figure 8). Moreover, the

209 washing solution was available for this device without damage, indicating that the material and

210 device are both applicable.

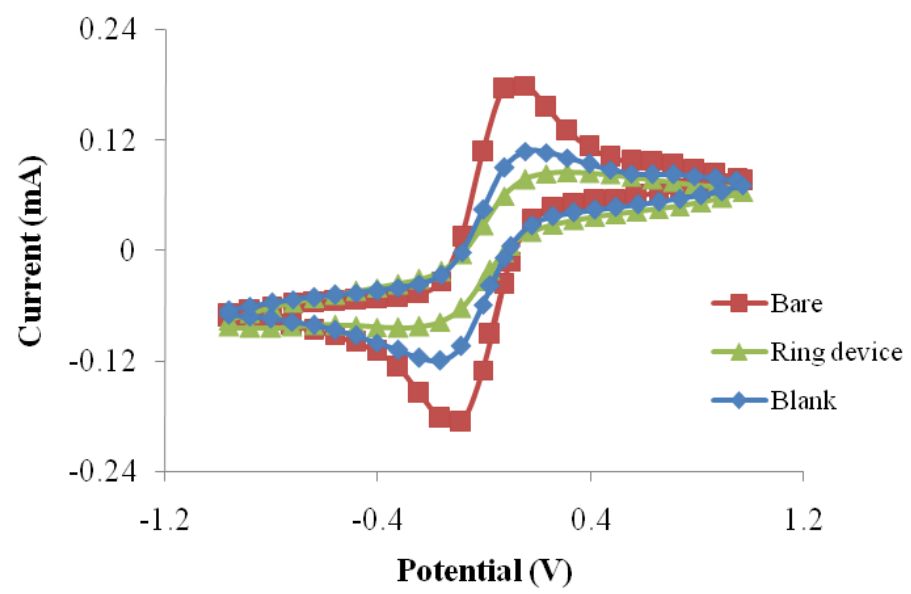

Figure 8. The CV performance of SPIMs with and without silica gel ring device.

\subsection{Performance of ring devices}


221 process. It can reduce the evaporation as well, which is advantageous because avoid the irregular

222 signal change can be avoided. Moreover, the devices can be recycled to decrease the detection

223 cost because of no damage after an easy nondestructive cleaning process.
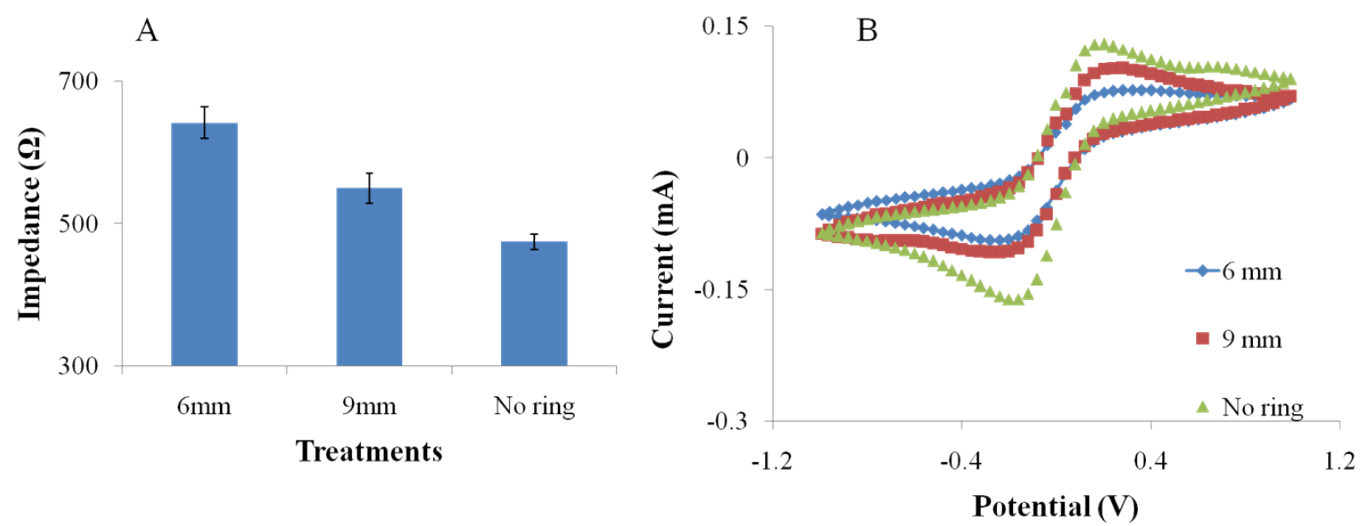

Figure 9. The EIS (A) and CV (B) performance of the SPIMs with and without 3D printed ring devices (6 and 9

226 millimeters).

\section{$227 \quad 3.5 \quad$ Base design using software}

229 reduce the irregular signal. In this paper, we designed two bases using software (Figure 10) that

230 the droplets can be reserved within the scope of detection area. We used 3D printer to print the

231 base, verifying the application (Figure 11). 
A
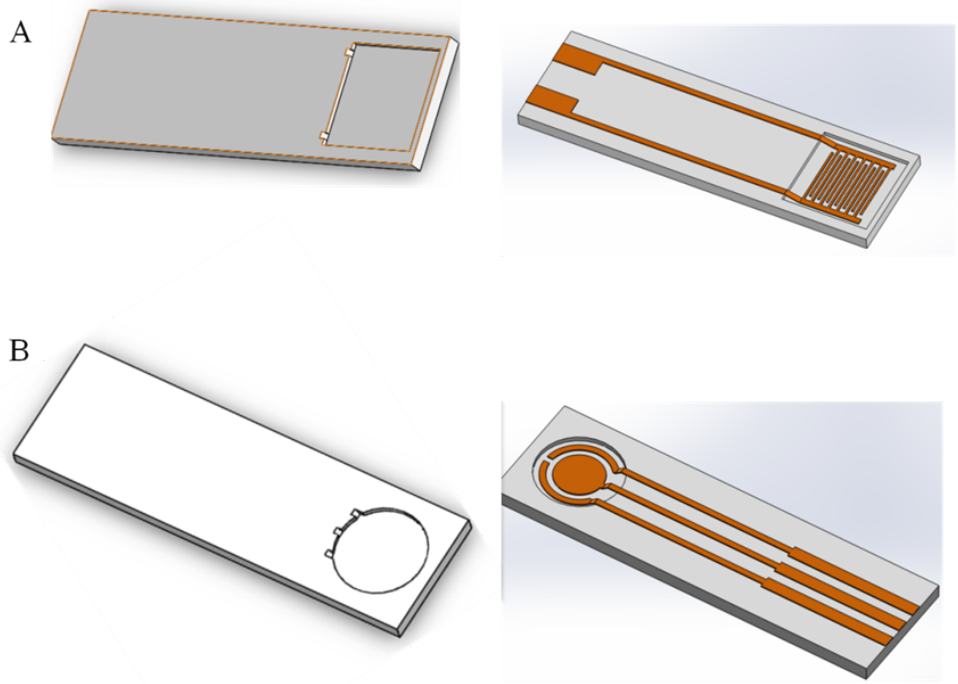

Figure 10. Two types of electrode bases designed by software. A) Interdigital microelectrode and B) Screenprinted electrode.

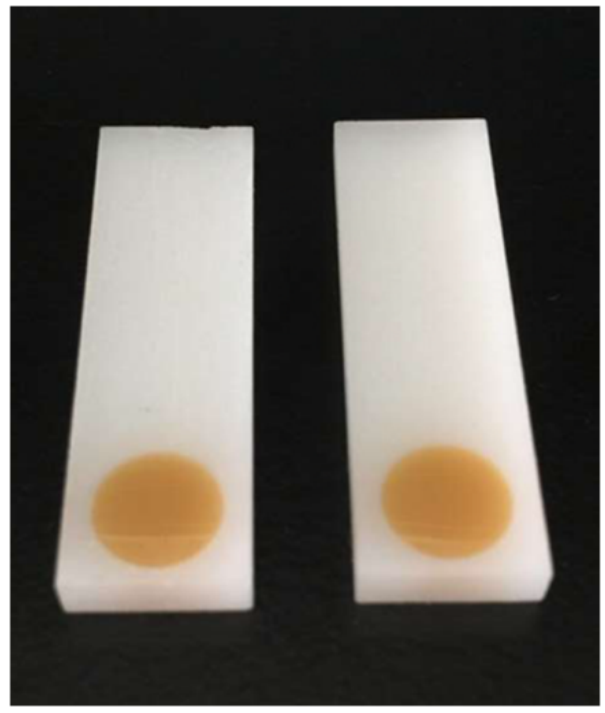

Figure 11.3D printed base was used to verify the application.

\section{Conclusion}

In the present research, SPIMs was employed to evaluate the influence of droplets coverage on the electrochemical response. 3D printing technology was used to print fabricate mini-small smart devices with different diameters based on nesting concept. Nest-like devices with different diameters $(2 \mathrm{r}=6,7,8,9$ millimeter $)$ were designed and printed to construct achieve different 
242

coverage to investigate the incubation performances and the results indicated that the influence of coverage on electrochemical response was significant. Moreover, ring devices on the surface of the SPIMs effectively improve the stability of the signal and also verify the such influence. Detection cost can be greatly reduced by recycling of the printed devices. All the devices improve the stability of the signal and eliminate the irregular signal change successfully. Our proposed design and concept show great potential for application in the field of electrodes fabrication and stable electrochemical biosensors construction.

\section{Acknowledgments}

The authors thank Biosensing \& Biomodeling Lab at Zhejiang University for their support of the electrodes and electrochemical station. We gratefully acknowledge Muyang Lin and Xi Yu (College of Chemistry, National University of Singapore) for their help.

\section{References}

Bernalte, E.; Sánchez, C.M.; Gil, E.P. 2011. Determination of mercury in ambient water samples by anodic stripping voltammetry on screen-printed gold electrodes. Anal. Chim. Acta, 689(1), 60-64.

Brett, C.M.A.; Thiemann, C. 2002. Conducting polymers from aminobenzoic acids and aminobenzenesulphonic acids: Influence of ph on electrochemical behaviour. J. Electroanal. Chem., 538-539, 215-222.

Ch, S.; Jenke, M.; Hoffmann, P.; Brugger, J. 2006. Interdigitated $50 \mathrm{~nm}$ ti electrode arrays fabricated using xef 2 enhanced focused ion beam etching. Nanotechnology, 17(11), 2722.

Costa, R.; Pereira, C.M.; Silva, A.F. 2015. Charge storage on ionic liquid electric double layer: The role of the electrode material. Electrochim. Acta, 167, 421-428.

Gerard, M.; Chaubey, A.; Malhotra, B.D. 2002. Application of conducting polymers to biosensors. Biosens. Bioelectron., 17(5), 345-359.

Gryczan, P.; Kisiel, A.; Michalska, A.; Maksymiuk, K. 2015. Electrochemical properties of polypyrrole doped by alternating polymer micelles. Electroanalysis, 27(3), 752-759.

Huang, X.; O'Mahony, A.M.; Compton, R.G. 2009. Microelectrode arrays for electrochemistry: Approaches to fabrication. Small, 5(7), 776-788.

Huey, E.; Krishnan, S.; Arya, S.K.; Dey, A.; Bhansali, S. 2012. Optimized growth and integration of silica nanowires into interdigitated microelectrode structures for biosensing. Sensors and Actuators B: Chemical, 
271

272

273

274

275

276

277

278

279

280

281

282

283

284

285

286

287

288

289

290

291

292

293

294

295

296

297

298

299

300

301

302

303

304

305

306

307

308

309

310

311

175, 29-33.

Hunt, H.K.; Armani, A.M. 2010. Label-free biological and chemical sensors. Nanoscale, 2(9), 1544-1559.

Kostecki, R.; Song, X.Y.; Kinoshita, K. 2000. Influence of geometry on the electrochemical response of carbon interdigitated microelectrodes. J. Electrochem. Soc., 147(5), 1878-1881.

Lepinay, S.; Staff, A.; Ianoul, A.; Albert, J. 2014. Improved detection limits of protein optical fiber biosensors coated with gold nanoparticles. Biosens. Bioelectron., 52, 337-344.

Li, Z.; Fu, Y.; Fang, W.; Li, Y. 2015a. Electrochemical impedance immunosensor based on self-assembled monolayers for rapid detection of escherichia coli o157:H7 with signal amplification using lectin. Sensors (Basel), 15(8), 19212-19224.

Li, Z.; Yu, Y.; Li, Z.; Wu, T. 2015b. A review of biosensing techniques for detection of trace carcinogen contamination in food products. Anal. Bioanal. Chem., 407(10), 2711-2726.

Liu, J.; Wagan, S.; Dávila Morris, M.; Taylor, J.; White, R.J. 2014. Achieving reproducible performance of electrochemical, folding aptamer-based sensors on microelectrodes: Challenges and prospects. Anal. Chem., 86(22), 11417-11424.

Olaru, A.; Bala, C.; Jaffrezic-Renault, N.; Aboul-Enein, H.Y. 2014. Surface plasmon resonance (spr) biosensors in pharmaceutical analysis. Crit. Rev. Anal. Chem., 45(2), 97-105.

Park, S.; Beskok, A. 2008. Alternating current electrokinetic motion of colloidal particles on interdigitated microelectrodes. Anal. Chem., 80(8), 2832-2841.

Santoro, C.; Guilizzoni, M.; Correa Baena, J.P.; Pasaogullari, U.; Casalegno, A.; Li, B.; Babanova, S.; Artyushkova, K.; Atanassov, P. 2014. The effects of carbon electrode surface properties on bacteria attachment and start up time of microbial fuel cells. Carbon, 67, 128-139.

Sun, J.; Zhou, W.; Huang, D.; Fuh, J.H.; Hong, G. 2015. An overview of 3d printing technologies for food fabrication. Food Bioprocess Technol, 8(8), 1605-1615.

Taleat, Z.; Khoshroo, A.; Mazloum-Ardakani, M. 2014. Screen-printed electrodes for biosensing: A review (2008-2013). Microchim. Acta, 181(9-10), 865-891.

Thévenot, D.R.; Toth, K.; Durst, R.A.; Wilson, G.S. 2001. Electrochemical biosensors: Recommended definitions and classification1. Biosens. Bioelectron., 16(1-2), 121-131.

Ueno, K.; Hayashida, M.; Ye, J.-Y.; Misawa, H. 2005. Fabrication and electrochemical characterization of interdigitated nanoelectrode arrays. Electrochem. Commun., 7(2), 161-165.

Valentini, F.; Ciambella, E.; Conte, V.; Sabatini, L.; Ditaranto, N.; Cataldo, F.; Palleschi, G.; Bonchio, M.; Giacalone, F.; Syrgiannis, Z.; Prato, M. 2014. Highly selective detection of epinephrine at oxidized single-wall carbon nanohorns modified screen printed electrodes (spes). Biosens. Bioelectron., 59, 94-98.

Van Dorst, B.; Mehta, J.; Bekaert, K.; Rouah-Martin, E.; De Coen, W.; Dubruel, P.; Blust, R.; Robbens, J. 2010. Recent advances in recognition elements of food and environmental biosensors: A review. Biosens. Bioelectron., 26(4), 1178-1194.

Vilé, G.; Albani, D.; Nachtegaal, M.; Chen, Z.; Dontsova, D.; Antonietti, M.; López, N.; Pérez - Ramírez, J. 2015. A stable single - site palladium catalyst for hydrogenations. Angewandte Chemie International Edition, 54(38), 11265-11269.

Wang, Y.; Ye, Z.; Ying, Y. 2012. New trends in impedimetric biosensors for the detection of foodborne pathogenic bacteria. Sensors, 12(3), 3449.

Wei, X.; Li, D.; Jiang, W.; Gu, Z.; Wang, X.; Zhang, Z.; Sun, Z. 2015. 3d printable graphene composite. 
312 Scientific Reports, 5, 11181.

313 Xing, J.; Zheng, M.; Duan, X. 2015. Two-photon polymerization microfabrication of hydrogels: An advanced

$3143 \mathrm{~d}$ printing technology for tissue engineering and drug delivery. Chem. Soc. Rev., 44(15), 5031-5039.

315 Xu, J.; Bao, N.; Xia, X.; Peng, Y.; Chen, H. 2004. Electrochemical detection method for nonelectroactive and 316 electroactive analytes in microchip electrophoresis. Anal. Chem., 76(23), 6902-6907.

317 Zhu, J.; Zhou, Y.; Gao, C. 1998. Influence of surfactants on electrochemical behavior of zinc electrodes in 318 alkaline solution. J. Power Sources, 72(2), 231-235. 